Check for updates

Cite this: Nanoscale Adv., 2020, 2, 401

\title{
Improvement in hole transporting ability and device performance of quantum dot light emitting diodes $\uparrow$
}

\author{
Pei-Chieh Chiu and Sheng-Hsiung Yang (D) *
}

In this research, we demonstrate a novel approach to improve the device performance of quantum dot light emitting diodes (QLEDs) by blending an additive BYK-P105 with poly(3,4ethylenedioxythiophene):polystyrene sulfonate (PEDOT:PSS) as the hole transport layer. In addition, for the first time, polyethylenimine ethoxylated (PEIE)-modified zinc oxide nanoparticles (ZnO NPs) as the electron transport layer were applied in regular-type QLEDs for achieving high device efficiency. A very high brightness of $139909 \mathrm{~cd} \mathrm{~m}^{-2}$ and current efficiency of $27.2 \mathrm{~cd} \mathrm{~A}^{-1}$ were obtained for the optimized device with the configuration of ITO/PEDOT:PSS + BYK-P105/PVK/CdSe QDs/ZnO NPs/PEIE/LiF/Al that shows promising use in light-emitting applications.

Received 2nd October 2019

Accepted 21st November 2019

DOI: 10.1039/c9na00618d

rsc.li/nanoscale-advances order to avoid this problem, Eric A. Meulenkamp proposed the synthesis of ZnO nanoparticles (NPs) with a diameter smaller than $10 \mathrm{~nm},{ }^{15}$ which were homogeneously dispersed in alcoholic solvents and requires only $150{ }^{\circ} \mathrm{C}$ to remove the solvent to form smooth $\mathrm{ZnO}$ thin films. L. Qian et al. demonstrated efficient QLEDs based on solution-processed multilayer structures. ${ }^{5}$ Their QLEDs were constructed with the configuration of indium tin oxide (ITO)/poly(3,4ethylenedioxythiophene):polystyrene sulfonate (PEDOT:PSS)/ poly[bis(4-phenyl)(4-butylphenyl)amine] (Poly-TPD)/CdSe QDs/ ZnO NPs/Al. The max brightness and power efficiency reached $68000 \mathrm{~cd} \mathrm{~m}^{-2}$ and $8.2 \mathrm{~lm} \mathrm{~W}^{-1}$, respectively, for green QLEDs.

Despite the rapid performance development in QLEDs, an intrinsic problem of electron-hole imbalance still exists. Compared with ZnO, the hole mobility of QLEDs is much lower and restricts their brightness and efficiency, ${ }^{10,16-19}$ mainly due to the adoption of organic hole transporting materials, such as PEDOT:PSS, Poly-TPD or poly[9,9-dioctylfluorene- $\mathrm{Co}-\mathrm{N}$-(4-butylphenyl)diphenylamine] (TFB). Therefore, many research groups attempt to improve charge balance with different solutions. It is reported that doping $\mathrm{Mg}^{2+}$ in ZnO NPs to form $\mathrm{ZnMgO}$ NPs slows down the electron mobility, which effectively solves the imbalance of carriers. ${ }^{\mathbf{1 0}, 16}$ Besides, the spherical ZnMgO NPs were found to be smaller than ZnO NPs that also modified interfacial properties between the ETL and CdSe QDs. The other method is to insert an electron blocking layer (EBL) between the active layer and the ETL. The EBL can not only effectively prohibit excessive electron injection into the QDs but also prevent the reverse transfer of electrons from CdSe QDs to the ETL, thereby preserving superior emissive efficiency. ${ }^{17-19} \mathrm{X}$. Dai et al. utilized poly(methyl methacrylate) (PMMA) as the EBL for the fabrication of QLEDs. ${ }^{17}$ The device structure was ITO/ PEDOT:PSS/Poly-TPD/poly( $N$-vinylcarbazole) (PVK)/CdSe QDs/
Institute of Lighting and Energy Photonics, College of Photonics, National Chiao Tung University, No. 301, Gaofa $3^{\text {rd }}$ Road, Guiren District, Tainan City 71150, Taiwan, Republic of China. E-mail: yangsh@mail.nctu.edu.tw; Fax: +886-6-3032535; Tel: +886-6-3032121 ext. 57895

$\dagger$ Electronic supplementary information (ESI) available. See DOI: 10.1039/c9na00618d 
PMMA/ZnO NPs/Ag and the optimized QLED achieved a high brightness of $42000 \mathrm{~cd} \mathrm{~m}^{-2}$ and external quantum efficiency (EQE) of $20.5 \%$. Q. Li's group synthesized a soluble tert-butyldimethylsilyl chloride-modified poly( $p$-phenylene benzobisoxazole) (TBS-PBO) as the EBL for simultaneous good balance of carrier transfer while maintaining a high current density. ${ }^{18}$ The luminance of the QLED with the configuration of ITO/ PEDOT:PSS/Poly-TPD/CdSe QDs/TBS-PBO/ZnO:Mg/Al achieved was $20000 \mathrm{~cd} \mathrm{~m}^{-2}$ and a max EQE of $16.7 \%$ was obtained. $\mathrm{H}$. Shen et al. proposed a different way to reduce hole injection barriers by choosing $\mathrm{ZnSe}$ as the shell material instead of commonly used $\mathrm{ZnS}$ or $\mathrm{CdS} .{ }^{20} \mathrm{~A}$ high record of max luminance of 356000614000 and $62600 \mathrm{~cd} \mathrm{~m}^{-2}$ was obtained for red, green and blue QLEDs. J. Song et al. modified ZnCdSe/ZnSe/ZnS QDs with different surface ligands to adjust the valence band (VB) from 6.48 and $6.21 \mathrm{eV}^{21}$ The constructed red QLED showed a brightness of $334000 \mathrm{~cd} \mathrm{~m}^{-2}$ and an EQE of up to $30.9 \%$.

Apart from ZnO NPs, an organic polymer, polyethylenimine ethoxylated (PEIE), has also been used as the ETL and a promising surface modifier in inverted QLEDs. H. H. Kim et al. claimed that PEIE can be easily incorporated on the surface of conducting metal oxides to reduce their work functions. ${ }^{22}$ They deposited a PEIE layer onto a colloidal ZnO NP film, enhancing electron injection into the active layer by lowering the work function of ZnO from $3.91 \mathrm{eV}$ to $2.91 \mathrm{eV}$. The red QLED with the configuration of ITO/ZnO NPs/PEIE/CdSe QDs/Poly-TPD:PVK/ $\mathrm{MoO}_{3} / \mathrm{Ag}$ showed a max brightness of $8600 \mathrm{~cd} \mathrm{~m}^{-2}$ and current efficiency of $1.53 \mathrm{~cd} \mathrm{~A}^{-1}$. Recently, Y. L. Shi et al. utilized PEIE to decrease the electron transporting barrier at the ITO/ZnO NPs and the electron injection barrier at the ZnO NPs/QD. ${ }^{23}$ The blue QLED with the configuration of ITO/PEIE/ZnO NPs/PEIE/coreshell ZnCdS-ZnS QDs/4,4'-bis( $N$-carbazolyl)-1,1'-biphenyl (CBP) $/ \mathrm{CBP}: \mathrm{MoO}_{3} / \mathrm{MoO}_{3} / \mathrm{Al}$ showed an increase in current efficiency from 2.25 to $3.1 \mathrm{~cd} \mathrm{~A}^{-1}$. In 2018, P. Shen et al. adopted $\mathrm{ZnO} / \mathrm{PEIE}$ as the ETL to fabricate white QLEDs. ${ }^{24}$ PEIE was incorporated to increase the conduction band (CB) of $\mathrm{ZnO}$, resulting in the blocking of electron injection to reach charge balance in QLEDs. A max brightness of $3554 \mathrm{~cd} \mathrm{~m}^{-2}$ and current efficiency of $10.5 \mathrm{~cd} \mathrm{~A}^{-1}$ were obtained for the device with the configuration of ITO/ZnO NPs/PEIE/RGB $(1: 1: 7)$ QDs/PVK/ PEDOT:PSS/Al. F. Cao et al. also selected PEIE to upshift the $\mathrm{CB}$ of $\mathrm{ZnO}$, thus blocking excess electrons into QDs to improve charge balance in QLEDs. ${ }^{25} \mathrm{~A}$ serially stacked white QLED with the configuration of ITO/ZnO NPs/PEIE/red QDs/PVK/ PEDOT:PSS/ZnO NPs/PEIE/green QDs/PVK/PEDOT:PSS/ZnO NPs/PEIE/blue QDs/PVK/PEDOT:PSS/Al was fabricated. An optimized QLED with a max brightness of $20320 \mathrm{~cd} \mathrm{~m}^{-2}$ and current efficiency of $79.9 \mathrm{~cd} \mathrm{~A}^{-1}$ was obtained. From the literature survey, we learned the fact that PEIE has only been utilized in inverted QLEDs, serving as the modifier for electrondominating oxide materials. To the best of our knowledge, PEIE has not been applied in regular-type QLEDs so far. This is the first report using PEIE as the surface modifier in regulartype QLEDs with enhanced brightness and efficiency.

As mentioned in the previous paragraph, the unbalanced carrier transport can be solved by the reduction of electron mobility or the addition of the EBL inside QLEDs as reported in the previous literature. In this research, we demonstrate a novel method to improve charge balance in QLEDs by modifying the hole mobility of the HTL. A controlled wetting and dispersing additive BYK-P105 for solvent-borne and solvent-free adhesives was discovered from commercial sources. It has a large acid value of $365 \mathrm{mg} \mathrm{KOH} \mathrm{per} \mathrm{g,} \mathrm{leading} \mathrm{to} \mathrm{a} \mathrm{strong} \mathrm{induced} \mathrm{effect} \mathrm{on}$ hole injection and transport. This unique additive has not been adopted for blending with PEDOT:PSS or other hole transporting materials to modify their properties so far. Hence, BYKP105 was selected to blend with PEDOT:PSS or PVK to augment hole mobility and to reach balanced charge transport in our QLEDs with improved device performance. From our experimental results, the addition of BYK-P105 in PEDOT:PSS is proven to increase hole transporting ability for QLED applications, which may also extend its potential use in other fields, such as organic light-emitting diodes, organic solar cells, and perovskite-related devices. Furthermore, a thin layer of PEIE was introduced on top of ZnO NPs to modify work functions in order to match the $\mathrm{LiF} / \mathrm{Al}$ electrode. In the early stage of this research, a common structure using ZnO NPs/Al for electron injection was studied and the resulting device performance is provided in Fig. S1 and Table S1 in the ESI. $\dagger$ A relatively high turn-on voltage of $5.3 \mathrm{~V}$ and much lower brightness as well as current efficiency were obtained for this simple QLED based on ZnO NPs/Al. By the incorporation of interfacial layers PEIE and LiF, the best QLED achieved a very high brightness of 139909 $\mathrm{cd} \mathrm{m}^{-2}$ and current efficiency of $27.2 \mathrm{~cd} \mathrm{~A}^{-1}$, with a low turn-on voltage of $3.8 \mathrm{~V}$. Our results reveal a new strategy for promoting the performance of QLEDs.

\section{Experimental section}

\subsection{Materials}

ITO glass substrates $\left(7 \Omega \square^{-1}\right.$ ) were purchased from Merck. PEDOT:PSS aqueous solution (Clevios P VP AI 4083) was purchased from Heraeus Precious Metals GmbH \& Co. KG. $37 \mathrm{wt} \%$ PEIE solution in water and PVK were purchased from Alfa Aesar and TCI, respectively. CdSe and additive BYK-P105 were provided by Opulence Optronics Co., Ltd. from Taiwan and BYK-Chemie $\mathrm{GmBH}$, respectively. Other reagents and solvents were bought from Alfa Aesar, Acros and ECHO Chemical Co., Ltd. and used without further purification.

\subsection{Preparation of ZnO NPs}

Colloidal ZnO NPs were synthesized using a sol-gel method. Zinc acetate dihydrate $(2.195 \mathrm{~g}, 10 \mathrm{mmol})$ and ethanolamine $(0.153 \mathrm{~g}, 2.5 \mathrm{mmol})$ were dissolved in $40 \mathrm{~mL}$ of dimethyl sulfoxide with vigorous stirring. Tetramethylammonium hydroxide (1.36 g, $7.5 \mathrm{mmol}$ ) was dissolved in $20 \mathrm{~mL}$ of isopropanol (IPA) with stirring and then dropped slowly to the zinc acetate solution within $10 \mathrm{~min}$ and continuously stirred for additional $30 \mathrm{~min}$. The above solution was poured into $300 \mathrm{~mL}$ of ethyl acetate and centrifuged at $3500 \mathrm{rpm}$ for $3 \mathrm{~min}$ to give a white precipitate. The supernatant was discarded and the white precipitate was dispersed in $n$-butanol to obtain $\mathrm{ZnO} \mathrm{NP}$ 
solution. The solution was filtered through a $0.45 \mu \mathrm{m}$ polytetrafluoroethylene filter before using.

\subsection{Device fabrication}

The light emitting devices with the regular configuration of ITO/ PEDOT:PSS/PVK/CdSe QDs/ZnO NPs/PEIE/LiF/Al were fabricated. The ITO substrates were cleaned sequentially with detergent, de-ionized water, acetone and IPA under ultrasonication for $30 \mathrm{~min}$, followed by nitrogen purge and ultraviolet-ozone treatment for $20 \mathrm{~min}$. The PEDOT:PSS without and with $1 \mathrm{wt} \%$ BYK-P105 was spin cast onto the ITO substrate at $3000 \mathrm{rpm}$ for $30 \mathrm{~s}$ and baked at $150{ }^{\circ} \mathrm{C}$ for $15 \mathrm{~min}$ in air. The substrates were then transferred into a nitrogen-filled glove box. PVK (in chlorobenzene, $8 \mathrm{mg} \mathrm{mL}^{-1}$ ) was spin coated on top of PEDOT:PSS at $3000 \mathrm{rpm}$ for $30 \mathrm{~s}$, followed by drying at $150{ }^{\circ} \mathrm{C}$ for 20 min. CdSe QDs (in $n$-octane, $12 \mathrm{mg} \mathrm{mL}^{-1}$ ) were spin coated onto the PVK layer at $2000 \mathrm{rpm}$ for $30 \mathrm{~s}$ and heated at $150{ }^{\circ} \mathrm{C}$ for $30 \mathrm{~min}$. ZnO NPs were deposited on top of the CdSe layer by spin coating at $2000 \mathrm{rpm}$ for $30 \mathrm{~s}$, followed by baking at $150{ }^{\circ} \mathrm{C}$ for 20 min. A thin layer of PEIE was deposited from its $0.4 \mathrm{wt} \%$ solution in 2-ethoxyethanol on ZnO NPs by spin coating at $5000 \mathrm{rpm}$ for $30 \mathrm{~s}$ and heated at $110{ }^{\circ} \mathrm{C}$ for $20 \mathrm{~min}$. Finally, $0.5 \mathrm{~nm}$ of $\mathrm{LiF}$ and $100 \mathrm{~nm}$ of aluminum electrodes were deposited by thermal evaporation under a base pressure of $\sim 10^{-6}$ Torr. The active area of each device is $1 \mathrm{~mm}^{2}$.

\subsection{Characterization methods}

The top-view and cross-sectional micrographs of the layer surface and devices were investigated with an ultrahigh resolution ZEISS Crossbeam scanning election microscope (SEM). The morphology and size of CdSe QDs were examined with a JEOL JEM-1400 transmission electron microscope (TEM). The surface morphology and roughness of CdSe QDs were performed using a Bruker Innova atomic force microscope (AFM). The absorption and photoluminescence (PL) spectra of samples were recorded with a Princeton Instruments Acton 2150 spectrophotometer equipped with a Xe lamp as the light source. The current density-voltage characteristics of hole- and electrononly devices were measured using an Agilent $4155 \mathrm{C}$ semiconductor parameter analyzer. The performance and electroluminescence spectra of QLEDs were recorded using an Agilent 4155C semiconductor parameter analyzer and an Ocean Optics USB2000 + spectrometer.

\section{Results and discussion}

The illustration of the QLED structure is shown in Fig. 1(a), which is constructed with the configuration of ITO/PEDOT:PSS/ PVK/CdSe QDs/ZnO NPs/PEIE/LiF/Al. The energy level diagram of the whole device is illustrated in Fig. 1(b). In our devices, we chose PEDOT:PSS as the hole injection material since its highest-occupied molecular orbital (HOMO) is very close to the work function of ITO. Next, PVK was adopted as the HTL between PEDOT:PSS and CdSe QDs. Like TFB and Poly-TPD, PVK is a well-known hole transporting material; besides, it shows good electron blocking ability because of the high-lying lowest-unoccupied molecular orbital (LUMO) level. Compared to TFB and Poly-TPD, PVK has a low-lying HOMO level $\left(\mathrm{HOMO}_{\mathrm{PVK}}=-5.8 \mathrm{eV},{ }^{17,26} \mathrm{HOMO}_{\mathrm{TFB}}=-5.3 \mathrm{eV},{ }^{8,10,19}\right.$ and $\mathrm{HOMO}_{\text {Poly-TPD }}=-5.2 \mathrm{eV}$ (ref. 16, 18 and 27)). The energy barrier between the HOMO of PVK and VB of CdSe QDs is the smallest and it induces more holes into the active layer and enhances carrier recombination. In addition, ZnO NPs with the CB of $-3.91 \mathrm{eV}$ was utilized as the ETL, while LiF/Al was evaporated as the electrode with a work function of $-2.8 \mathrm{eV}^{28}$ In order to match the energy level between the ETL and LiF/Al electrode, an amino-based hydrophilic polymer, PEIE, was incorporated to reduce the CB of ZnO NPs. The CB of PEIE-modified ZnO was reported to be $-2.9 \mathrm{eV},{ }^{20}$ which beneficially led to a smaller energy barrier to inject more electrons from the $\mathrm{LiF} / \mathrm{Al}$ electrode into the ETL.

Fig. 2(a) shows the TEM image of CdSe QDs with an average diameter of $10 \mathrm{~nm}$. To examine the film-forming properties and surface roughness, two characterization techniques, SEM and AFM, were carried out in this research. The SEM micrograph of the CdSe layer spincast from its $n$-octane solution is shown in Fig. 2(b), revealing a featureless surface. The AFM topographic image of the CdSe layer is displayed in Fig. 2(c) and the corresponding average surface roughness is measured to be only $1.74 \mathrm{~nm}$. Such a highly covered and evenly distributed active layer helps to reduce leakage current that has a positive effect on (a)

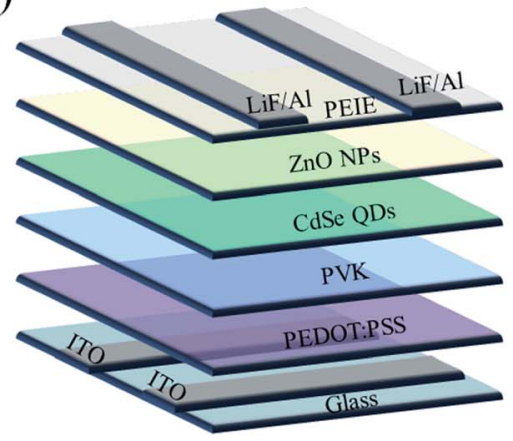

(b)

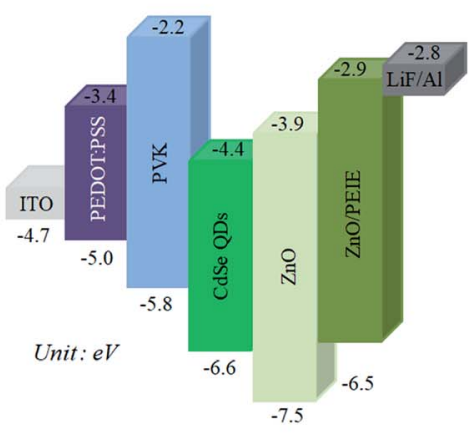

Fig. 1 (a) Device structure and (b) energy level diagram of the QLEDs. 

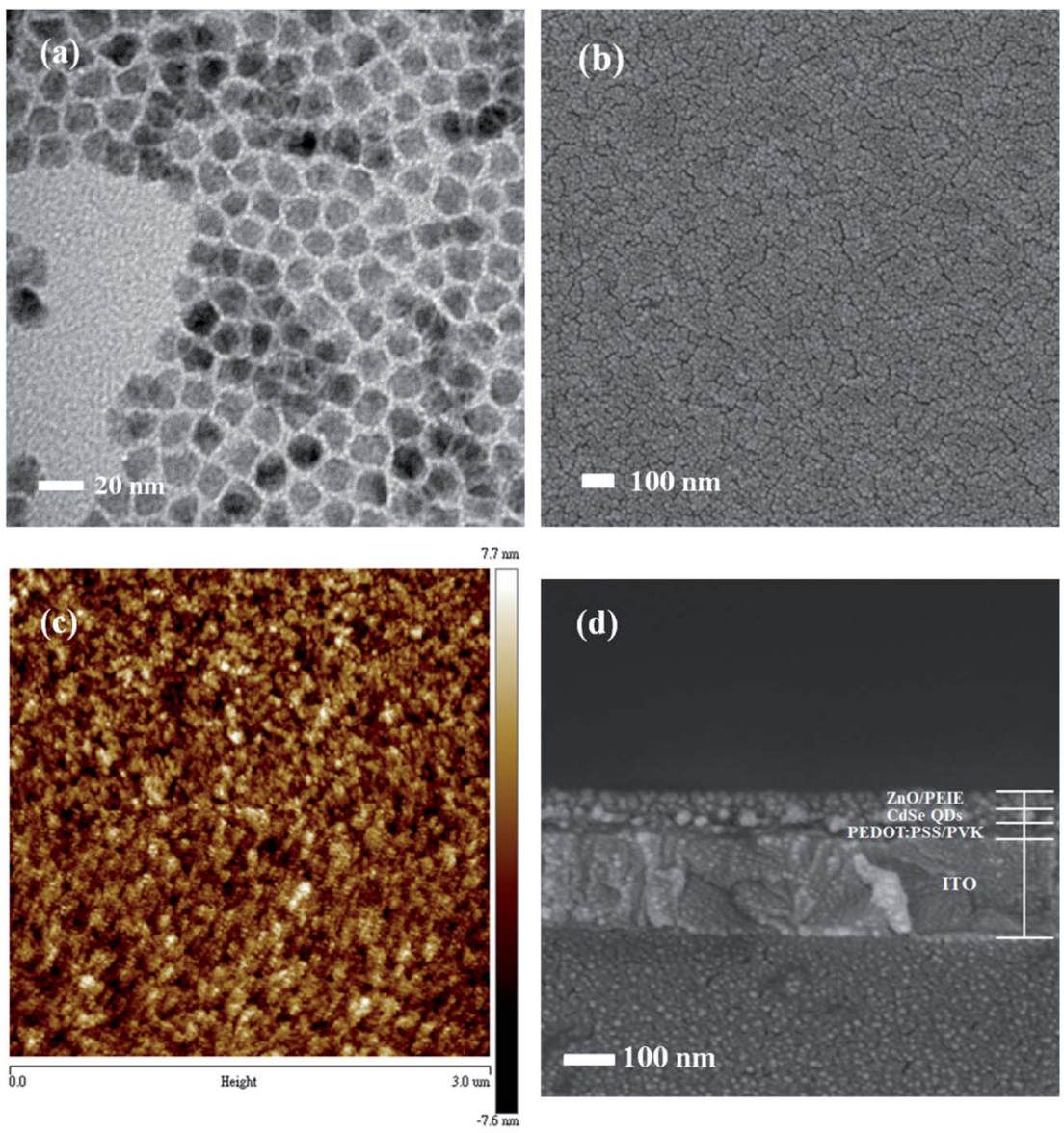

Fig. 2 (a) TEM, (b) SEM and (c) AFM images of CdSe QDs; (d) cross-sectional SEM image of the whole device.

device performance. The SEM cross-sectional image of the whole device is shown in Fig. 2(d). The thicknesses of the PEDOT:PSS/PVK, CdSe QDs, and ZnO/PEIE are estimated to be 45,35 , and $45 \mathrm{~nm}$, respectively. The UV-vis absorption and PL emission spectra of CdSe QDs in the solution state are shown in Fig. 3. The optical bandgap of $2.21 \mathrm{eV}$ was estimated from its absorption edge in Fig. 3. A pure green light with an emission peak centered at $539 \mathrm{~nm}$ and a full-width at half-maximum (FWHM) of $31 \mathrm{~nm}$ were obtained. Several literature studies on green light CdSe QDs and their QLEDs have been reported with emission wavelength from 507 to $540 \mathrm{~nm}$ and FWHM from 33 to $38 \mathrm{~nm},{ }^{3,6,29}$ depending on the $\mathrm{S} / \mathrm{Se}$ composition ratio in the core. It is seen that CdSe QDs utilized in this study possess a narrower bandwidth compared with other literature reports.

To exploit a new device structure for CdSe QDs, four different QLEDs were fabricated for comparison, and the corresponding current density-voltage, brightness-voltage, current efficiencycurrent density and EQE-voltage characteristics are shown in Fig. 4(a)-(d). The structure of the standard device I is ITO/ PEDOT:PSS/PVK/CdSe QDs/ZnO NPs/LiF/Al. The maximum brightness, current efficiency and EQE were measured to be $58985 \mathrm{~cd} \mathrm{~m}^{-2}, 9.22 \mathrm{~cd} \mathrm{~A}^{-1}$ and $2.08 \%$, respectively. For device II, a thin layer of PEIE was deposited on the ZnO NPs for the first time in the development of regular-type QLEDs. We found that the performance of device II with PEIE was dramatically increased, reaching $98563 \mathrm{~cd} \mathrm{~m}^{-2}, 26.9 \mathrm{~cd} \mathrm{~A}^{-1}$ and 6.09\%, which are much higher than those of device I. The reason for this phenomenon can be attributed to the lowered CB of ZnO NPs by PEIE and enhanced electron injection from the LiF/Al electrode. In order to further improve device brightness and current efficiency, an additive BYK-P105 from the commercial source was blended with either PVK or PEDOT:PSS to improve the device performance. The structure of device III is ITO/

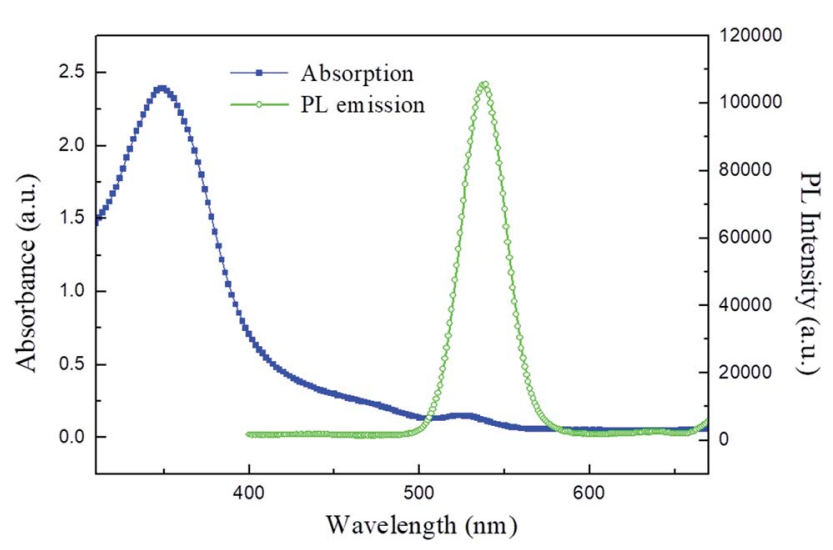

Fig. 3 UV-vis absorption and PL emission spectra of CdSe QDs. 

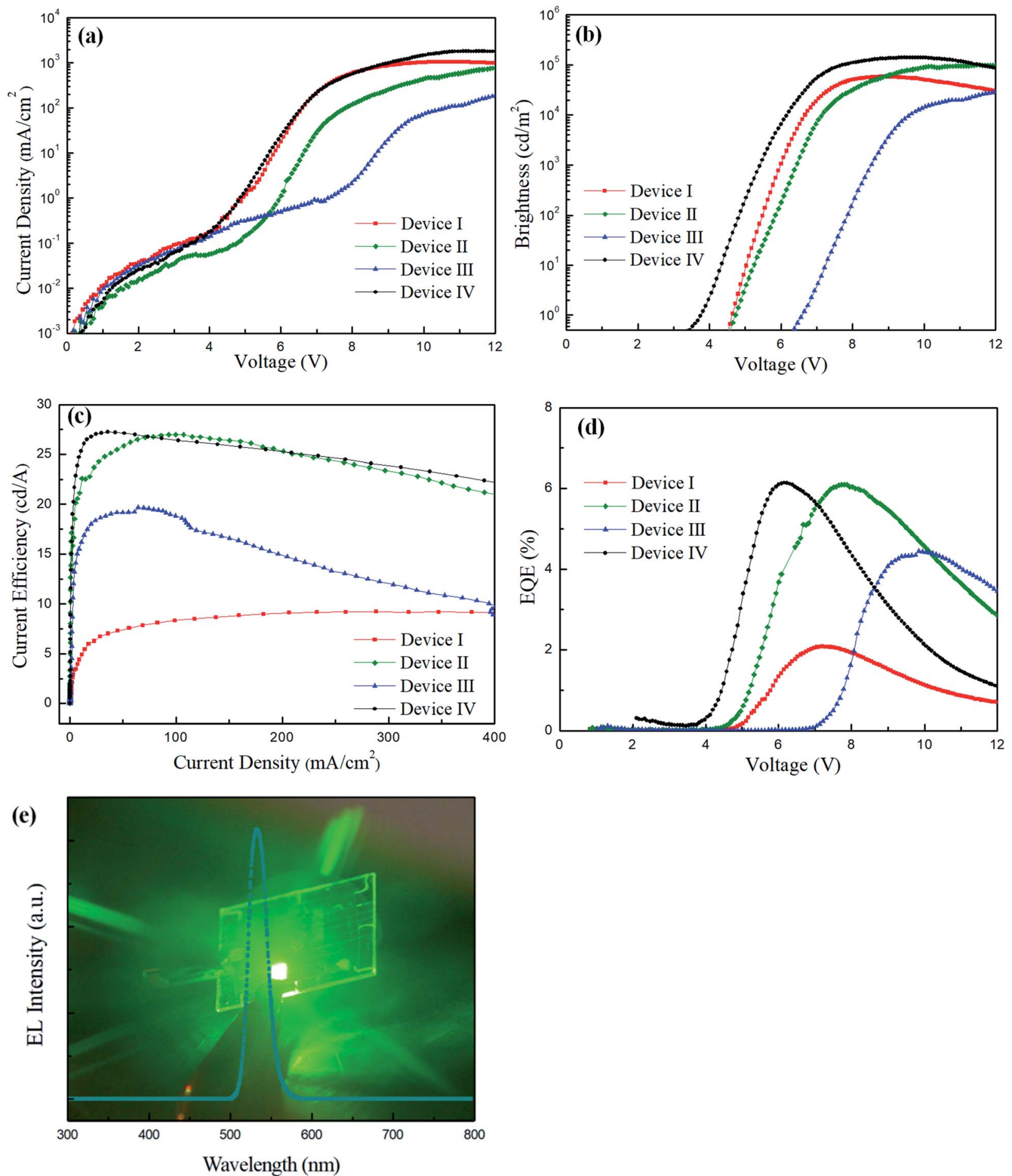

Fig. 4 (a) Current density-voltage, (b) brightness-voltage, (c) current efficiency-current density and (d) EQE-voltage characteristics of the four QLEDs; (e) EL spectrum and snapshot of driving device IV.

PEDOT:PSS/PVK + BYK-P105/CdSe QDs/ZnO NPs/PEIE/LiF/Al, while the structure of device IV is ITO/PEDOT:PSS + BYKP105/PVK/CdSe QDs/ZnO NPs/PEIE/LiF/Al. By blending BYKP105 with PVK, the maximum luminance, current efficiency and EQE of device III were measured to be $39737 \mathrm{~cd} \mathrm{~m}^{-2}, 19.6$ cd $\mathrm{A}^{-1}$ and $4.44 \%$, respectively, which were significantly reduced compared with device II. By blending BYK-P105 with PEDOT:PSS, the maximum luminance and current efficiency of device IV were surprisingly increased up to $139909 \mathrm{~cd} \mathrm{~m}^{-2}, 27.2$ cd $\mathrm{A}^{-1}$ and $6.14 \%$, respectively, and the turn-on voltage was notably decreased to $3.8 \mathrm{~V}$. It is seen that the addition of $1 \mathrm{wt} \%$ BYK-P105 into different HTLs brought large difference in device 
Table 1 Device performance of the four QLEDs with different structures

\begin{tabular}{|c|c|c|c|c|}
\hline Device & $\begin{array}{l}\text { Turn-on } \\
\text { voltage }^{a}(\mathrm{~V})\end{array}$ & $\begin{array}{l}\text { Max brightness } \\
{\left[\mathrm{cd} \mathrm{m}^{-2} @ \mathrm{QV}\right]}\end{array}$ & $\begin{array}{l}\text { Max current efficiency } \\
{\left[\mathrm{cd} \mathrm{A}^{-1} \text { @ }\right]}\end{array}$ & Max EQE [\% @V] \\
\hline I & 4.6 & 58985 @8.85 & $9.22 @ 7.2$ & $2.08 @ 7.2$ \\
\hline III & 6.6 & 39737 @13.8 & 19.6 @9.8 & 4.44 @9.8 \\
\hline IV & 3.8 & 139909 @9.8 & 27.2 @6.15 & 6.14 @6.15 \\
\hline
\end{tabular}

${ }^{a}$ Defined as the operating voltage when the brightness reached $1 \mathrm{~cd} \mathrm{~m}^{-2}$.

performance. Besides, we also prepared 0.5 wt $\%$ BYK-P105 in PEDOT:PSS solution to examine its concentration effect. The device performance from $0.5 \mathrm{wt} \%$ BYK-P105 has been provided in Fig. S2 and Table S2 in the ESI. $\dagger$ It is seen that the brightness and current efficiency of the QLED were lower than those of the one using $1 \mathrm{wt} \%$ BYK-P105. By increasing the concentration to 2 wt\%, however, BYK-P105 was not totally dissolved in PEDOT:PSS as observed with the naked eye. We observed that PEDOT:PSS blended with 1 wt\% BYK-P105 and showed good film-forming properties since both of them possess an ionic structure. On the contrary, the structural nature of PVK is quite different from that of the additive BYK-P105 that affects miscibility in the solution state. The formed composite film was not very uniform and thus carrier transport ability was prohibited. The top-view SEM images of the two HTLs before and after blending with BYK-P105 are provided in Fig. S3 in the ESI. $\dagger$ It is seen that PEDOT:PSS retained a good film state before and after blending with BYK-P105; however, the PVK film blended with BYK-P105 showed some pinholes and hence the surface of the substrate was not fully covered. The electroluminescence (EL) spectrum of device IV is shown in Fig. 4(e), revealing very bright green light with a maximum EL wavelength at $532 \mathrm{~nm}$. All device performance from the four different QLEDs is summarized in Table 1. The comparison of the device performance of green QLEDs from the previous literature studies and our study is listed in Table $2 .^{4-6,10,20,24,25,27,29,30}$ It is seen that our optimized device IV comprising PEDOT:PSS + BYK-P105 as the HTL and PEIE-modified ZnO as the ETL showed a very high brightness and comparable current efficiency compared with other studies. Therefore, blending BYK-P105 with PEDOT:PSS provides a successful approach to improve the performance of QLEDs.
In order to confirm the enhanced hole transport capability of PEDOT:PSS by adding BYK-P105 and to verify whether charge balance is achieved, two hole-only ITO/PEDOT:PSS/Al and ITO/ PEDOT:PSS + BYK-P105/Al devices and one electron-only ITO/ ZnO NPs/PEIE/LiF/Al device were fabricated to measure their current-voltage characteristics, as shown in Fig. 5 . The device based on PEDOT:PSS + BYK-P105 showed a higher current value, implying better hole transport than the pristine PEDOT:PSS one. The hole mobility of the PEDOT:PSS before and after blending with BYK-P105 was obtained from the spacecharge limited current model $J=(9 / 8) \varepsilon_{\mathrm{r}} \varepsilon_{0} \mu_{\mathrm{e}} V^{2} / d^{3}$, assuming $\varepsilon_{\mathrm{r}}=$

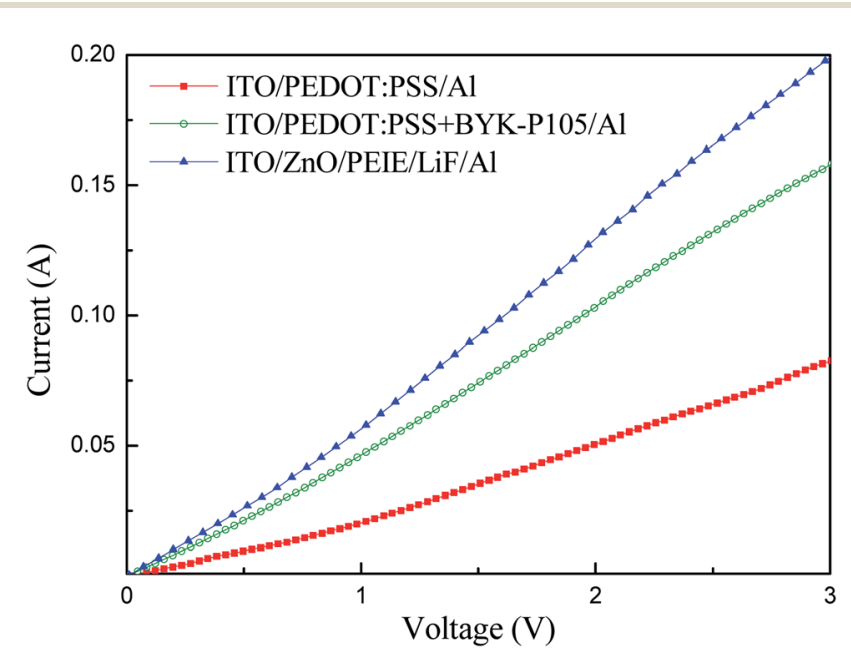

Fig. 5 Current-voltage characteristics of the hole-only and electrononly devices.

Table 2 Comparison of green QLED performance based on different device structures

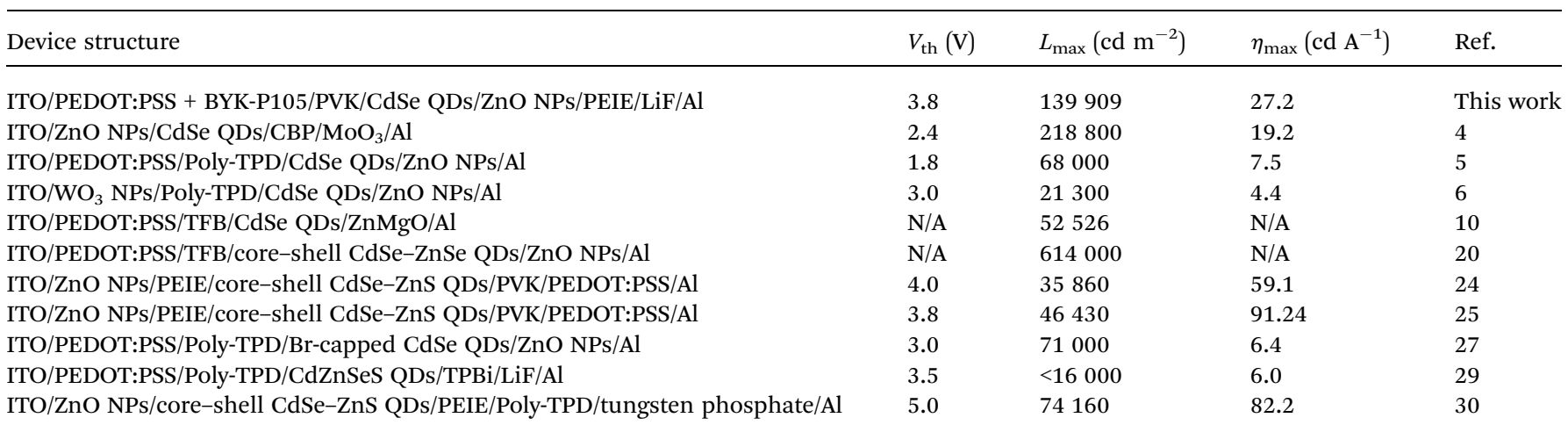


$4,^{10,17} \varepsilon_{0}=8.854 \times 10^{-12} \mathrm{~F} \mathrm{~m}^{-1}$, and film thickness $d=45 \mathrm{~nm}$. The hole mobility of PEDOT:PSS and PEDOT:PSS + BYK-P105 films was estimated to be $1.13 \times 10^{-4}$ and $2.686 \times 10^{-4} \mathrm{~cm}^{2}$ $\mathrm{V}^{-1} \mathrm{~s}^{-1}$, respectively, revealing an enhanced value by using BYKP105. Moreover, the conductivity of the PEDOT:PSS + BYK-P105 device was closer to that of the ZnO NPs/PEIE device, indicating more balanced carrier transport that is responsible for the improved device performance, as shown in Fig. 4 and Table 1.

\section{Conclusions}

In this research, we successfully fabricated high-efficiency QLEDs by blending BYK-P105 with PEDOT:PSS to modify the hole transport. Meanwhile, for the first time, PEIE-modified ZnO NPs were utilized as the ETL to achieve carrier balance in regular-type QLEDs. The best device exhibited a maximum brightness of $139909 \mathrm{~cd} \mathrm{~m}^{-2}$ and current efficiency of $27.2 \mathrm{~cd}$ $\mathrm{A}^{-1}$ and shows promising use in light-emitting applications.

\section{Conflicts of interest}

The authors declare that there is no conflict of interest.

\section{Acknowledgements}

The authors thank the Ministry of Science and Technology of Republic of China (grant number MoST 107-2221-E-009-042MY3) for financial support of this work.

\section{References}

1 P. O. Anikeeva, J. E. Halpert, M. G. Bawendi and V. Bulovic, Nano Lett., 2009, 9, 2532-2536.

2 S. Coe, W. K. Woo, M. Bawendi and V. Bulovic, Nature, 2002, 420, 800-803.

3 Y. Yang, Y. Zheng, W. Cao, A. Titov, J. Hyvonen, J. R. Manders, J. Xue, P. H. Holloway and L. Qian, Nat. Photonics, 2015, 9, 259-266.

4 J. Kwak, W. K. Bae, D. Lee, I. Park, J. Lim, M. Park, H. Cho, H. Woo, D. Y. Yoon, K. Char, S. Lee and C. Lee, Nano Lett., 2012, 12, 2362-2366.

5 L. Qian, Y. Zheng, J. g Xue and P. H. Holloway, Nat. Photonics, 2011, 5, 543-548.

6 X. Yang, Y. Ma, E. Mutlugun, Y. Zhao, K. S. Leck, S. T. Tan, H. V. Demir, Q. Zhang, H. Du and X. W. Sun, ACS Appl. Mater. Interfaces, 2014, 6, 495-499.

7 K. S. Cho, E. K. Lee, W. J. Joo, E. Jang, T. H. Kim, S. J. Lee, S. J. Kwon, J. Y. Han, B. K. Kim, B. L. Choi and J. M. Kim, Nat. Photonics, 2009, 3, 341-345.

8 J. Pan, J. Chen, Q. Huang, Q. Khan, X. Liu, Z. Tao, Z. Zhang, W. Lei and A. Nathan, ACS Photonics, 2016, 3, 215-222.
9 B. S. Mashford, T. L. Nguyen, G. J. Wilsonb and P. Mulvaney, J. Mater. Chem., 2010, 20, 167-172.

10 S. Wang, Y. Guo, D. Feng, L. Chen, Y. Fang, H. Shen and Z. Du, J. Mater. Chem. C, 2017, 5, 4724-4730.

11 M. N. Kamalasanan and S. Chandra, Thin Solid Films, 1996, 288, 112-115.

12 E. Redel, P. Mirtchev, C. Huai, S. Petrov and G. A. Ozin, ACS Nano, 2011, 5, 2861-2869.

13 A. K. K. Kyaw, X. W. Sun, C. Y. Jiang, G. Q. Lo, D. W. Zhao and D. L. Kwong, Appl. Phys. Lett., 2008, 93, 221107.

14 D. Zheng, G. Wang, W. Huang, B. Wang, W. Ke, J. L. Logsdon, H. Wang, Z. Wang, W. Zhu, J. Yu, M. R. Wasielewski, M. G. Kanatzidis, T. J. Marks and A. Facchetti, Adv. Funct. Mater., 2019, 29, 1900265.

15 E. A. Meulenkamp, J. Phys. Chem. B, 1998, 102, 5566-5572. 16 Q. Zhang, X. Gu, Q. Zhang, J. Jiang, X. Jin, F. Li, Z. Chen, F. Zhao and Q. Li, Opt. Mater. Express, 2018, 8, 909-918.

17 X. Dai, Z. Zhang, Y. Jin, Y. Niu, H. Cao, X. Liang, L. Chen, J. Wang and X. Peng, Nature, 2014, 515, 96-99.

18 X. Jin, C. Chang, W. Zhao, S. Huang, X. Gu, Q. Zhang, F. Li, Y. Zhang and Q. Li, ACS Appl. Mater. Interfaces, 2018, 10, 15803-15811.

19 D. Li, J. Bai, T. Zhang, C. Chang, X. Jin, Z. Huang, B. Xu and Q. Li, Chem. Commun., 2019, 55, 3501-3504.

20 H. Shen, Q. Gao, Y. Zhang, Y. Lin, Q. Lin, Z. Li, L. Chen, Z. Zeng, X. Li, Y. Jia, S. Wang, Z. Du, L. S. Li and Z. Zhang, Nat. Photonics, 2019, 13, 192-197.

21 J. Song, O. Wang, H. Shen, Q. Lin, Z. Li, L. Wang, X. Zhang and L. S. Li, Adv. Funct. Mater., 2019, 29, 1808377.

22 H. H. Kim, S. Park, Y. Yi, D. I. Son, C. Park, D. K. Hwang and W. K. Choi, Sci. Rep., 2015, 5, 8968.

23 Y. L. Shi, F. Liang, Y. Hu, M. P. Zhuo, X. D. Wang and L. S. Liao, Nanoscale, 2017, 9, 14792-14797.

24 P. Shen, X. Li, F. Cao, X. Ding and X. Yang, J. Mater. Chem. C, 2018, 6, 9642-9648.

25 F. Cao, D. Zhao, P. Shen, J. Wu, H. Wang, Q. Wu, F. Wang and X. Yang, Adv. Opt. Mater., 2018, 6, 1800652.

26 J. Pan, J. Chen, Q. Huang, Q. Khan, X. Liu, Z. Tao, W. Lei, F. Xua and Z. Zhang, RSC Adv., 2015, 5, 82192-82198.

27 B. H. Kang, J. S. Lee, S. W. Lee, S. W. Kim, J. W. Lee, S. A. Gopalan, J. S. Park, D. H. Kwon, J. H. Bae, H. R. Kim and S. W. Kang, Sci. Rep., 2016, 6, 34659.

28 W. K. Bae, J. Kwak, J. W. Park, K. Char, C. Lee and S. Lee, Adv. Mater., 2009, 21, 1690-1694.

29 W. K. Bae, K. Char, H. Hur and S. Lee, Chem. Mater., 2008, 20, 531-539.

30 F. Cao, Q. Wu and X. Yang, ACS Appl. Mater. Interfaces, 2019, 11, 40267-40273. 